\title{
Bogoliubov Fermi surface revealed
}

\author{
Discovery of segmented Fermi surface induced by Cooper pair momentum \\ Authors: Zhen Zhu, Michał Papaj, Xiao-Ang Nie, Hao-Ke Xu, Yi-Sheng Gu, Xu \\ Yang, Dandan Guan, Shiyong Wang, Yaoyi Li, Canhua Liu, Jianlin Luo, Zhu-An Xu, \\ Hao Zheng, Liang Fu, and Jin-Feng Jia \\ arXiv:2010.02216
}

\section{Recommended with a Commentary by Carlo Beenakker, Instituut-Lorentz, Leiden University}

The Fermi surface of a metal separates electronic states that are occupied at zero temperature from states that remain empty. It is obtained by solving for zero excitation energy, $E(\boldsymbol{p})=0$, to produce a $d-1$ dimensional surface in $d$-dimensional momentum space. Can a superconductor have a Fermi surface, a surface of zero excitation energy for Bogoliubov quasiparticles? That question was addressed theoretically by Volovik many years ago, and an experimental demonstration is now reported by Zhu et al. (arXiv:2010.02216).

A figure from Volovik's 2006 paper (arXiv:cond-mat/0601372) explains the mechanism for the emergence of a Bogoliubov Fermi surface. The excitation gap $\Delta$ closes if the velocity $v_{s}$ of the Cooper pairs exceeds a critical velocity $v_{c}=\Delta / p_{\mathrm{F}}$. This is the Doppler effect first pointed out for superfluids by Landau, which shifts the quasiparticle energy by an amount

$$
\delta E(\boldsymbol{p})=\boldsymbol{p} \cdot \boldsymbol{v}_{s}
$$

The gap remains open for momentum directions $p_{\perp}$ perpendicular to the superflow, producing the segmented Fermi surface shown in the figure - provided that $v_{s}$ remains below the depairing velocity $v_{*}$ at which the superconductor becomes a normal metal.

In a bulk superconductor $v_{*} \approx v_{c}$ so Volovik's mechanism is not operative, but if superconductivity is induced by the proxim-

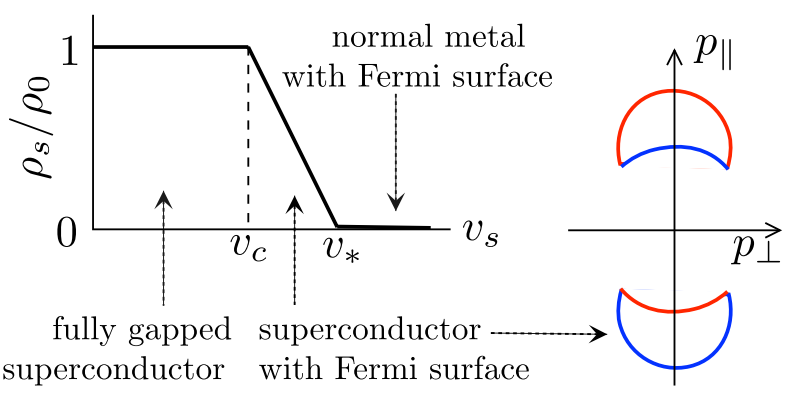

Upon increasing the velocity $v_{s}$ of the superconducting condensate, the excitation gap closes at a critical velocity $v_{c}=\Delta / p_{\mathrm{F}}$ and a Bogoliubov Fermi surface appears. The left panel shows the drop in the Cooper pair density $\rho_{s}$, which vanishes at the depairing velocity $v_{*}$. The right panel shows the contours of zero excitation energy: a Fermi surface formed out of electron-like states (red) and hole-like states (blue) appears for $v_{c}<v_{s}<v_{*}$. [Adapted from Volovik (2006).] ity effect in a $2 \mathrm{D}$ electron gas one may have $v_{c}=\Delta_{\text {induced }} / p_{\mathrm{F}}$ small compared to the depairing velocity in the bulk. This is the approach 
taken by Zhu et al., following up on a proposal by Yuan and Fu (arXiv:1801.03522). The $2 \mathrm{D}$ electron gas on the surface of the topological insulator $\mathrm{Bi}_{2} \mathrm{Te}_{3}$ is proximitized by the superconductor $\mathrm{NbSe}_{2}$. An in-plane magnetic field $B$ induces a screening supercurrent over a London penetration depth $\lambda$, which boosts the Cooper pair momentum by an amount $p_{s} \simeq e B \lambda$, in-plane and perpendicular to $B$. The proximity induced gap is reduced below the bulk gap, and thus only a small magnetic field is needed to significantly affect the quasiparticle spectrum without strongly impacting the parent superconductor.

The 2D electrons on the surface of a 3D topological insulator are massless Dirac fermions, which requires a modification of the usual expression (1) for the Doppler shift of massive Schrödinger electrons. In the ideal case of an isotropic dispersion, $\varepsilon(\boldsymbol{p})=v_{\mathrm{F}}|\boldsymbol{p}|$, the Doppler shift due to a Cooper pair momentum $p_{s}$ is given by

$$
\delta E(\boldsymbol{p})=\frac{v_{\mathrm{F}}}{|\boldsymbol{p}|} \boldsymbol{p} \cdot \boldsymbol{p}_{s}
$$

For $\mathrm{Bi}_{2} \mathrm{Te}_{3}$ the dispersion relation is anisotropic,

$$
\varepsilon(\boldsymbol{p})=v_{\mathrm{F}} \sqrt{|\boldsymbol{p}|^{2}+\alpha^{2} p_{x}^{2}\left(p_{x}^{2}-3 p_{y}^{2}\right)^{2}},
$$

and a more general expression is needed: ${ }^{1}$

$$
\delta E(\boldsymbol{p})=\frac{\partial \varepsilon(\boldsymbol{p})}{\partial \boldsymbol{p}} \cdot \boldsymbol{p}_{s} .
$$

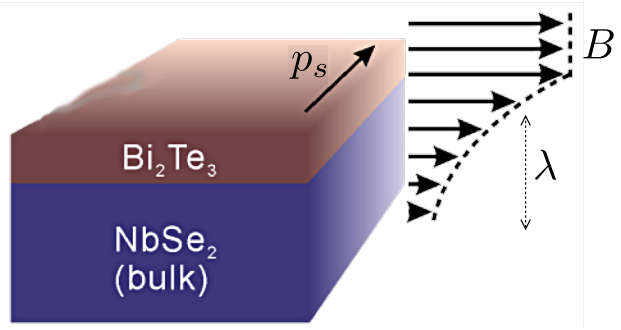

Heterostructure studied by Zhu et al. The $\mathrm{Bi}_{2} \mathrm{Te}_{3}$ topological insulator film is sufficiently thin that the $\mathrm{NbSe}_{2}$ superconductor can induce a pair potential in the $2 \mathrm{D}$ electron gas on the top surface. An in-plane magnetic field $B$ gives a nonzero Cooper pair momentum $p_{s} \simeq e B \lambda$.

The corresponding critical momentum depends on the direction of the supercurrent,

$$
p_{c}= \begin{cases}\Delta / v_{\mathrm{F}} & \text { in the } y \text {-direction, } \\ \left(\Delta / v_{\mathrm{F}}\right)\left(1-\frac{5}{2} \alpha^{2} p_{\mathrm{F}}^{4}\right) & \text { in the } x \text {-direction. }\end{cases}
$$

To observe the Bogoliubov Fermi surface Zhu et al. use the technique of FourierTransform Scanning Tunneling Microscopy (Petersen et al., 1998). Surface electrons scattered by defects produce an oscillatory interference pattern in the local density of states (Friedel oscillations), with a spatial periodicity set by the difference of wave vectors on the Fermi surface. The Fourier transform of the spatial $d I / d V$ map measured at low bias voltages with a scanning probe reveals these periodicities.

\footnotetext{
${ }^{1}$ Eq. (4) holds to first order in $\boldsymbol{p}_{s}$ for a time-reversally-symmetric single-electron Hamiltonian $H_{0}(\boldsymbol{p})$ and an $s$-wave pair potential $\Delta$. The Cooper pair momentum enters in the Bogoliubov-De Gennes Hamiltonian $\mathcal{H}(\boldsymbol{p})=H_{0}(\boldsymbol{p}) \tau_{z}+\Delta \tau_{x}$ as an offset $\boldsymbol{p} \mapsto \boldsymbol{p}+\tau_{z} \boldsymbol{p}_{s}$, with $\tau_{z}$ a Pauli matrix in the electron-hole degree of freedom. The linearized energy shift is $\delta E=\tau_{z} \boldsymbol{p}_{s} \cdot\langle\partial \mathcal{H} / \partial \boldsymbol{p}\rangle=\tau_{0} \boldsymbol{p}_{s} \cdot\left\langle\partial H_{0} / \partial \boldsymbol{p}\right\rangle=\tau_{0} \boldsymbol{p}_{s} \cdot \partial\left\langle H_{0}\right\rangle / \partial \boldsymbol{p}$, in view of Hellmann-Feynman and $\tau_{z}^{2}=\tau_{0}$. Because $\tau_{0} H_{0}$ and $\mathcal{H}$ commute, they can be jointly diagonalized and the expectation value $\left\langle H_{0}\right\rangle$ in the basis of eigenstates of $\mathcal{H}$ is equal to an eigenvalue $\varepsilon(\boldsymbol{p})$ of $H_{0}(\boldsymbol{p})$, hence $\delta E=\boldsymbol{p}_{s} \cdot \partial \varepsilon / \partial \boldsymbol{p}$. I have not found this simple formula in the literature and thank Yaroslav Herasymenko for the derivation.
} 
Zhu et al. present a detailed numerical analysis of their experimental data, but the key features are evident upon inspection: In the absence of a magnetic field the hexagonally warped Fermi surface of Eq. (3) is visible in the $d I / d V$ map for voltages outside of the superconducting gap, while at $V=0$ the $d I / d V$ map is featureless. Application of an in-plane magnetic field reintroduces the segments of the Fermi surface with wave vectors in the perpendicular direction.

Volovik's supercurrent mechanism is not the only way to create an extended Bogoliubov Fermi surface (a gapless $d-1$ dimensional manifold in $d$-dimensional momentum space). In a multiband superconductor with a nodal pair potential the pairing of mismatched Fermi surfaces can expand a nodal point or nodal line into a $2 \mathrm{D}$ surface seg-

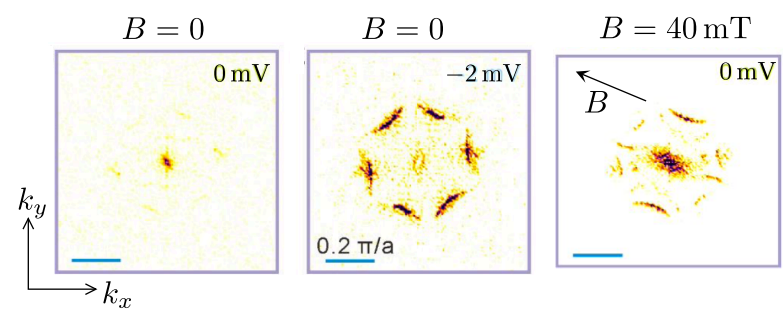

Fourier transformed $d I / d V$ maps, measured at different bias voltages $V$ and magnetic fields $B$. At $B=0$ the hexagonal Fermi contour of the $\mathrm{Bi}_{2} \mathrm{Te}_{3}$ surface electrons is visible outside of the superconducting gap $\left(e|V|=2 \mathrm{meV}>\Delta_{\text {induced }}=0.5 \mathrm{meV}\right)$, while it vanishes inside the gap. An in-plane magnetic field of $40 \mathrm{mT}$ closes the gap in the perpendicular direction. Only the electron-like segments of the Bogoliubov Fermi surface are probed by the STM for $V=0^{+}$.

ment. This mechanism could be operative in a $d_{x^{2}-y^{2}}$ superconductor in a Zeeman field (Yang and Sondhi, 1998) or in a chiral pair potential with intrinsically broken time-reversal symmetry (Agterberg, Brydon, and Timm, 2017; Link and Herbut, 2020). I am not aware of any experimental demonstration along these lines.

The simplicity of the realization of Zhu et al. promises much follow-up work. Papaj and $\mathrm{Fu}$ (arXiv:2006.06651) propose to confine the gapless superconducting state to a narrow 1D channel, surrounded by 2D regions with a full superconducting gap. Majorana bound states may emerge at the end points of the channel, providing an alternative platform to existing semiconductor nanowire based systems. 